\title{
Conceptual frameworks of synthetic lethality in clear cell carcinoma of the ovary (Review)
}

\author{
HIROSHI KOBAYASHI, NAOKI KAWAHARA, KENJI OGAWA, YUKI YAMADA, \\ KANA IWAI, EMIKO NIIRO and SACHIKO MORIOKA
}

Department of Obstetrics and Gynecology, Nara Medical University, Nara 634-8522, Japan

Received April 27, 2018; Accepted June 15, 2018

DOI: $10.3892 /$ br.2018.1114

\begin{abstract}
Targeting non-oncogenes may result in the selective death of cancer cells. Clear cell carcinoma of the ovary (CCC) may exhibit resistance against conventional chemotherapy and is associated with poor prognosis. The aim of the present report was to review synthetic lethality-based therapies for CCC. Previous English-language studies were reviewed to accumulate preclinical and clinical data on targeting synthetic lethal partners. Synthetic lethal interactions have a variety of types, involving components of a backup or parallel pathway with overlapping functions, components encoded by paralogous pairs, subunit components that form heteromeric complexes and components that are arranged in a single linear pathway. A set of candidate gene targets potentially resulting in synthetic lethality have been previously identified. HNF class homeobox, AT-rich interaction domain 1A, ATR serine/threonine kinase, ATM serine/threonine kinase, checkpoint kinase 1 and phosphatase and tensin homolog may be the key partner genes. A variety of loss of function genes in CCC are driver or passenger events and may function as synthetic lethal pairs under replication stress conditions. Further clinical studies will be required to investigate the safety and therapeutic effect of synthetic lethality pairs in CCC tumor types with replication stress.
\end{abstract}

\section{Contents}

1. Introduction

2. Literature search

3. Mutations and molecular heterogeneity of ovarian cancer

4. Synthetic lethality

5. Synthetic lethal partners in $\mathrm{CCC}$

6. Conclusions

Correspondence to: Professor Hiroshi Kobayashi, Department of Obstetrics and Gynecology, Nara Medical University, 840 Shijo-cho, Kashihara, Nara 634-8522, Japan

E-mail: hirokoba@naramed-u.ac.jp

Key words: synthetic lethality, ovarian cancer, clear cell carcinoma, genetic mutations, homologous recombination

\section{Introduction}

Ovarian cancer is the seventh most common cancer among women globally (1). Clinicians are facing an increasing number of ovarian cancer cases diagnosed each year. This disease is associated with a poor prognosis as the majority of cases are diagnosed at an advanced stage (1). Clinical observations and molecular analyses have divided ovarian cancer into two major subtypes: Type 1 cancer types, which are composed of low grade serous cancer, endometrioid cancer, clear cell cancer and mucinous cancer; and type 2 cancer types, which include high grade serous cancers (HGSC), carcinosarcomas and undifferentiated carcinomas (2). Clear cell and endometrioid carcinomas arise in endometriosis [endometriosis-associated ovarian cancer (EAOC)] (2). HGSC was originally thought to develop from the ovarian surface epithelium and/or ovarian inclusion cysts (2). However, recent research has contradicted this and suggests that the majority of HGSC develops from fallopian tube fimbrial epithelium (3).

Recent promising advances in the field of cancer treatment have resulted in the development of successful strategies for molecular-targeted medicine (including small-molecule inhibitors and antibodies), clinical applications of immunotherapy and identification of synthetic lethal partners (4). Substantial progress has been made in the treatment of ovarian cancer through use of targeted therapies and immunotherapy (5). Anti-angiogenic therapy using bevacizumab, considered the most promising targeted therapy for ovarian cancer, may improve the overall survival time of patients with poor prognosis (6). One previous study also demonstrated the safety and antitumor activity of nivolumab, an anti-programmed cell death protein 1 (PD-1) antibody that blocks PD-1 signaling, in patients with platinum-resistant ovarian cancer (7). Since observations have questioned the prospect of using PD-ligand 1 (PD-L1) expression as a biomarker for discriminating responders from non-responders, it must be complemented by a superior predictive biomarker. Such a predictive biomarker must be able to select sensitive patients in order to reduce toxicity and costs and increase progression-free survival and overall survival time. In addition, resistance to therapy is a major problem due to molecular heterogeneity (4). The prioritized driver or passenger genes, including p53, BRCA1/2, DNA repair associated (BRCA1/2), AT-rich interaction domain 1A (ARID1A), phosphatase and tensin homolog (PTEN), phos- 


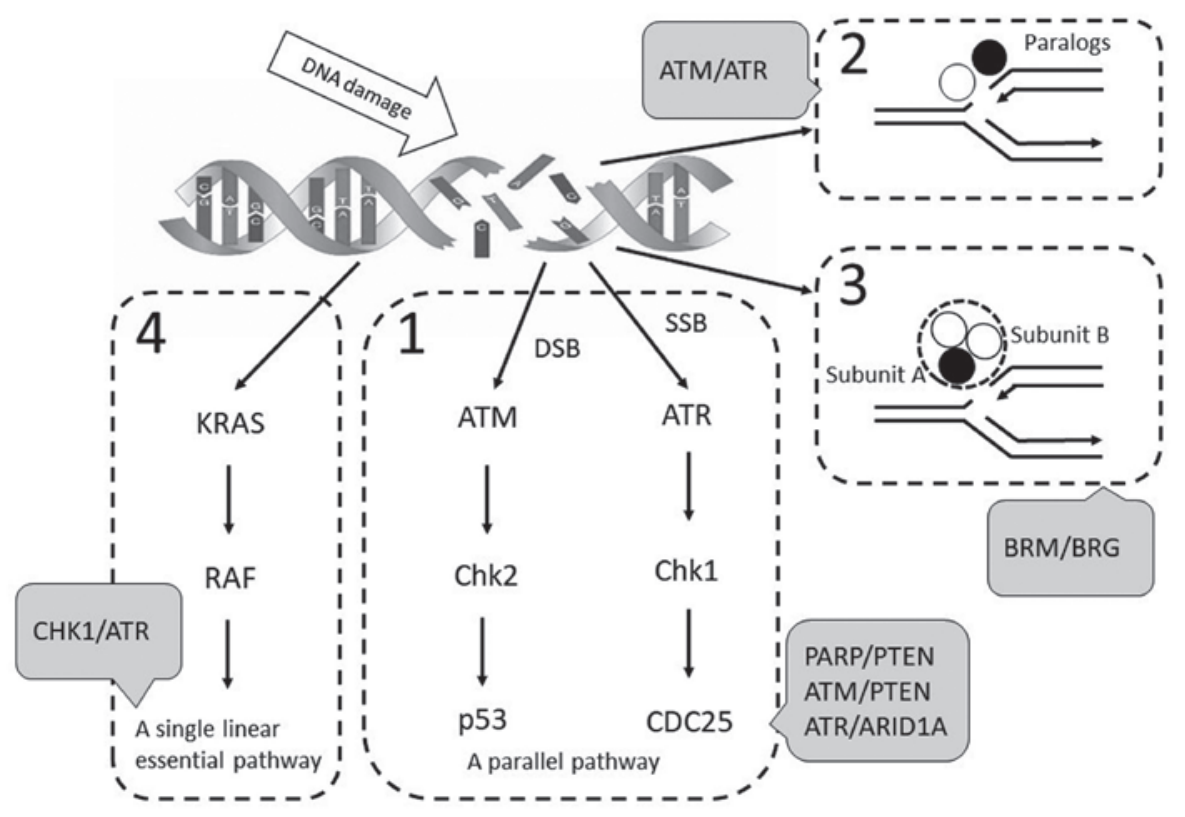

Figure 1. Landscape of synthetic lethal pairs. Synthetic lethal pairs were divided into four categories: (1) Components of a backup or a parallel pathway with overlapping functions; (2) components encoded by paralogous pairs; (3) Subunit components that form heteromeric complexes; and (4) components that are arranged in a single linear pathway. KRAS, KRAS proto-oncogene, GTPase; Chk, checkpoint kinase; ATR, ATR serine/threonine kinase; ATM, ATM serine/threonine kinase; p53, tumor protein p53; PARP, poly(ADP-ribose) polymerases; PTEN, phosphatase and tensin homolog; ARID1A, AT-rich interaction domain 1A; BRM, SWItch/Sucrose non-fermentable-related, matrix associated, actin dependent regulator of chromatin, subfamily a, member 2; BRG, SWItch/Sucrose non-fermentable-related, matrix associated, actin dependent regulator of chromatin, subfamily a, member 4 .

phatidylinositol-4,5-bisphosphate 3-kinase catalytic subunit $\alpha$ (PIK3CA) and MYC proto-oncogene, bHLH transcription factor (c-MYC), exert loss- or gain-of-functions (4). Functional losses in tumor suppressor genes cannot be restored through small molecules or specific antibodies (8). Furthermore, the functions of intracellular oncogene products, including Ras and c-MYC, may be difficult to modulate directly due to the increasing cytotoxicity of normal cells (8).

The aim of the present report was to provide a review of synthetic lethality-based therapies for patients with ovarian cancer. Additionally, future perspectives on their plausible applications for novel therapeutic methods of clear cell carcinoma of the ovary (CCC) in the era of personalized medicine are provided.

\section{Literature search}

A computerized literature search was conducted to identify relevant studies reported in the English language. MEDLINE submissions (https://www.ncbi.nlm.nih.gov/pubmed) were searched for studies published between January 2000 and April 2018, combining the keywords 'ovarian cancer', 'ovarian clear cell carcinoma', 'carcinogenesis, 'synthetic lethality' and 'DNA damage repair'. A variety of combinations of these terms were used, depending on which database was searched. Each gene was also linked to the associated National Centre for Biotechnology Information Entrez Gene pages (http://www.ncbi.nlm.nih.gov/sites/entrez). Furthermore, the references of each article were searched to identify potentially relevant studies. Publications of original studies and review papers were included, while those documenting opinions, points of view or anecdotes were discarded.

\section{Mutations and molecular heterogeneity of ovarian cancer}

HGSC has been frequently associated with loss-of-function genes [including tumor protein p53 (p53), BRCA1/2, cyclin dependent kinase inhibitor $2 \mathrm{~A}$ also known as $\mathrm{p} 16^{\mathrm{INK} 4 \mathrm{a}}, \mathrm{RB}$ transcriptional corepressor 1 and PTEN] (3). Previous genomic study has indicated that germline and somatic mutations in homologous recombination genes including BRCA1/2 and p53 may increase the risk of HGSC (9). Homologous recombination is essential for DNA damage repair, prevention of various mutations and maintenance of genomic integrity (10). HGSC tumor types also exhibit somatic loss of function of BRCA and its associated genes (3). Certain sporadic cancer types that share the molecular features of BRCA1/2-mutant tumor types may respond to similar therapeutic strategies (11). This trait is referred to as 'BRCAness'. Furthermore, the key oncogenic driver, c-MYC, and the G1/S phase regulatory gene, cyclin E (CCNE1), are frequently amplified in HGSC and are major factors in tumorigenesis (3). MYC-amplified cancer cells have been associated with elevated levels of DNA replication stress (12). Amplification of CCNE1 and mutation of BRCA1/2 genes are reportedly mutually exclusive in HGSC, suggesting that CCNE1 induces synthetic lethality in BRCA1/2-mutated cells (3).

Oxidative stress induced by repeated hemorrhaging increases the susceptibility of endometriotic cells to DNA damage and subsequent malignant transformation, resulting in the development of type 1 cancer, named EAOC (13). Type 1 cancer has a high mutational burden: Somatic gene mutations identified include those in ARID1A mutations (40-50\%), PIK3CA (40\%), PTEN (20\%), catenin beta 1 (CTNNB1; $16-54 \%)$, protein phosphatase 2 scaffold subunit $\alpha(20 \%)$, 
KRAS proto-oncogene, GTPase (KRAS; 4-5\%), microsatellite instability (13-50\%) and overexpression of transcription factor hepatocyte nuclear factor-1 $\beta$ (HNF; $>90 \%$ in CCC) (14). ARID1A is the most frequent driver mutation in EAOC (15). PIK3CA/protein kinase $\mathrm{B} /$ mechanistic target of rapamycin (mTOR) pathway is also commonly altered in CCC (16). Furthermore, the overexpression of HNF has been identified in a large proportion of CCC (17). Checkpoint kinase 1 (Chk1) has been revealed to be a downstream target of HNF and serve a role within the cell cycle checkpoint pathways (18). DNA damage increases the persistent phosphorylation of Chk1 and induction of $\mathrm{G} 2 / \mathrm{M}$ phase cell cycle arrest in cells overexpressing $\operatorname{HNF}(19,20)$. HNF deletion, in turn, induces apoptosis, suggesting that enhanced levels of HNF may be essential for survival in CCC $(17,20)$. Molecular changes, including those in HNF and Chk1, may be a manifestation of essential alterations in cell cycle regulation, detoxification and chemoresistance in CCC $(13,18,21)$. Targeted therapy with specific inhibitors of Chk1 or the HNF-Chk1 axis may be explored as a potential treatment modality for patients with CCC (22). This knowledge may result in novel potential combination therapeutic methods, for instance chemotherapeutic agents and targeting of Chk1, which may overcome drug resistance and produce more successful disease treatment. In this regard, a number of important drivers have been identified in ovarian cancer, including BRCA1/2, p53, CCNE1, ARID1A, Chk1 and HNF. These alterations represent potential future therapeutic opportunities for CCC.

\section{Synthetic lethality}

The concept of tumor-selective synthetic lethality as a therapeutic strategy is as follows: Two genes are 'synthetically lethal' if concurrent loss of the two genes results in cellular lethality or cell death, whereas the deficiency of either alone is compatible with cellular viability (8). Specific inhibitors of the cancer driver genes or oncogenes may contribute to development of idiosyncratic toxicity (23). An example of synthetic lethality is PARP inhibition in BRCA1/2-deficient ovarian and/or breast cancer types $(3,24,25)$. Since homologous recombination-associated genes, including BRCA1, BRCA2, RAD51 recombinase (RAD51), Fanconi anemia complementation group and p53 genes, are essential for DNA damage repair and survival, cancer cells may use an alternative approach (non-oncogenes) and become dependent upon the parallel route (26). Therefore, targeting non-oncogenes may result in the selective death of the cancer cells (27). If one gene, one paralog or one pathway is inactive (e.g., germline BRCA mutations), the loss or inhibition of a second functionally parallel gene or pathway (e.g., PARP inhibitions) may result in cell death specifically (26). Pharmacological inhibition of PARP alone may selectively kill homologous recombination deficient cancer cells but leave normal cells intact (26).

Synthetic lethality screening. The identification of synthetic lethal interactions is made possible by the SynLethDB database (http://histone.sce.ntu.edu.sg/SynLethDB/) (28). A comprehensive understanding of the synthetic lethality-related genes and their corresponding interactions is essential for developing novel therapeutics against cancer using network-based research
Table I. List of candidate synthetic lethality gene pairs those with germline and/or somatic mutation in four categories.

Components of a backup or a parallel pathway with overlapping functions.

\begin{tabular}{lc}
\hline PARP & BRCA \\
PARP & CDK12 \\
PARP & PTEN \\
PARP & ATM \\
PARP & Cohesin \\
mTOR & TP53 \\
VEGFR2 & TP53 \\
EGFR & TP53 \\
CSNK1E & TP53 \\
CTNNB1 & TP53 \\
CSNK1A1 & TP53 \\
CDK6 & TP53 \\
CSNK1A1 & APC \\
TDO2 & APC \\
CTNNB1 & APC \\
BRAF & APC \\
IGF1R & APC \\
WNK1 & APC \\
ATM & ATR \\
ATM & TP53 \\
ATM & PTEN \\
ATM & PRKCA \\
ATR & XRCC1 \\
ATR & TOP1 \\
ATR & POLD1 \\
ATR & ARID1A \\
CDK1 & KRAS \\
MAPK14 & KRAS \\
PRKDC & MYC \\
CSNK1E & MYC \\
CKS1B & PTEN \\
KRAS & PLK1 \\
TRRAP & PLK1 \\
HH & PIK3CA \\
HGS & PIK3CA \\
NLK & CTNNB1 \\
POLB & \\
POLD1 & \\
\hline & \\
\hline &
\end{tabular}

Components encoded by paralogous pairs.

$\begin{array}{lc}\text { ATM } & \text { ATR } \\ \text { TRRAP } & \text { PIK3CA } \\ \text { CBP } & \text { EP300 } \\ \text { BMR } & \text { BRG }\end{array}$


Table I. Continued.

Subunit components that form heteromeric complexes.

\begin{tabular}{lc}
\hline RAD52 & BRCA \\
PARP1 & RAD54B \\
PARP1 & RAD51 \\
BMR & BRG \\
\hline
\end{tabular}

Components that are arrangedin a single linear pathway.

\begin{tabular}{lc}
\hline Chk1 & ATR \\
RAF1 & KRAS \\
\hline
\end{tabular}

A synthetic lethal pair (left gene) of germline and/or somatic mutation gene (right gene).

platforms (29). The high mutational burden of cancer cells may contain genes that trigger susceptibility to synthetic lethality and are synthetic lethal interactors that indicate a therapeutic opportunity (30). Despite the high number of mutated genes and the consequential plethora of potential gene-gene interaction data in CCC, identifying synthetically lethal genes and their partners is challenging due to the difficulty of distinguishing true partners from errors (31). Genomic high-throughput experimental data may aid investigators to understand the synthetic lethal screening, including from high-throughput forward genetic screening approaches, genome-wide small interfering RNA (siRNA) or clustered regularly interspaced short palindromic repeat-based human cell line screening, and comprehensive in silico pipeline computational approaches, or a combination of these methods (32). Among these screenings, the dominant approach is high-throughput screening using RNA interference or compound libraries (32). At present, a kinome siRNA library of $>40,000$ siRNAs targeting $>10,000$ genes (four siRNAs per gene) is commercially available. In a previous study, a variety of human cancer cell lines with a particular tumor suppressor gene or oncogene were plated in arrayed siRNA library-coated wells using a library targeting protein networks (8). The hit list from the primary screen was then systematically narrowed. Candidate synthetic lethality genes were validated to confirm whether synthetic lethal pairs selectively kill cancer cells, but not normal cells (8). However, limitations for the screening-based approach are a high false positive rate of computational predictions and high cost.

Synthetic lethal gene pairs. In the present review, the synthetic lethal pairs were divided into four categories as described by Fang (8): i) Components of a backup or a parallel pathway with overlapping functions; ii) components encoded by paralogous pairs; iii) subunit components that form heteromeric complexes and iv) components that are arranged in a single linear pathway (Fig. 1).

Category 1: Components of a backup or a parallel pathway with overlapping functions. Mutational screening studies of synthetic lethality have been reported (8,33-36). The majority of the category 1 genes are associated with DNA damage repair and cell survival. A high number of DNA double-strand breaks are repaired by BRCA-dependent homologous recombination (37). PARP1 may serve as an alternative or a backup pair (38). Preclinical studies revealed that proteins involved in DNA repair and response to damage, including BRCA1/2, X-ray repair cross complementing (XRCC)1, XRCC2, XRCC3, ATM serine/threonine kinase (ATM), ATR serine/threonine kinase (ATR), Chk1, Chk2, proliferating cell nuclear antigen, RAD51, MRE11 homolog, double strand break repair nuclease, ERCC excision repair 1, endonuclease non-catalytic subunit, p53, cyclin dependent kinase 12 (CDK12) and PTEN, in addition to the Fanconi anemia pathway, induce synthetic lethality with PARP inhibitors and are implicated as potential predictive markers for tumor cell response to PARP inhibitors $(8,33)$. As presented in Table I, PARP inhibitors (Table I; A synthetic lethal pair) are currently under investigations as therapeutic agents for the treatment of cancer types with deficiencies in BRCA, CDK12, PTEN, ATM, ATR or Cohesin genes (Table I; Germline and/or somatic mutations) $(34,35)$. Furthermore, comprehensive experiments have identified novel synthetic lethal gene pairs, including p53-ATM, p53-ATR, TP53-mTOR, p53-vascular endothelial growth factor receptor 2, p53-epidermal growth factor receptor, p53-transforming acidic coiled-coil containing protein 3, p53-MYC, p53-CTNNB1, p53-casein kinase 1 epsilon (CSNK1E), ATR-ARID1A, ATR-XRCC1, ATR-DNA topoisomerase I, ATR-DNA polymerase $\delta 1$, catalytic subunit, ATM-protein kinase $\mathrm{C} \alpha$, KRAS-CDK1, KRAS-polo like kinase 1 (PLK1), CDC28 protein kinase regulatory subunit 1-PLK1, APC-CTNNB1, APC-casein kinase $1 \alpha 1$, APC-tryptophan 2,3-dioxygenase, CSNK1E-MYC, CTNNB1-hepatocyte growth factor-regulated tyrosine kinase substrate, PTEN-nemo like kinase and mutS homolog 2-DNA polymerase beta $(32,34,36)$.

Category 2: Components encoded by paralogous pairs (paralogs). DNA damage response pathway factors, including ATM and ATR, result in a parallel signaling cascade that is activated by replication stress and uses a complex and coordinated set of proteins to maintain genomic stability (39). ATR is considered among the top candidates of the category 2 genes, as ATR inhibitors are entering clinical trials and have therapeutic utility (34). cAMP-response element binding protein (CREBBP) serves a role in growth by coupling chromatin remodeling to transcription factor recognition (40). The CREBBP paralog, p300/EP300, is a synthetic-lethal gene in CREBBP-deficient cancer types (41). SWItch/Sucrose non-fermentable (SWI/SNF)-related, matrix associated, actin dependent regulator of chromatin, subfamily a, member 2 (BRM) and SWI/SNF-related, matrix associated, actin dependent regulator of chromatin, subfamily a, member 4 (BRG) are DNA-dependent ATPases of the SWI/SNF chromatin remodeling complex (42). Synthetic lethality may be explained by BRM or BRG paralog insufficiency (42). Two pair genes are organized in the networks of paralogs (42).

Category 3: Subunit components that form heteromeric complexes. The SWI/SNF complex facilitates the homologous recombination-dependent processes, including the recruitment of BRM, BRG, ARID and RAD molecules to DNA double-strand breaks (43). In a similar manner to PARP1 
inhibitors, the suppression of RAD52 reduces homologous recombination and results in synthetic lethality in cells deficient in BRCA1/2 or associated proteins (including partner and localizer of BRCA2, RAD51B/C/D and XRCC2/3), whilst sparing normal cells (44). RAD51/RAD54B and PARP1 have been reported to function as synthetic lethal interactors, where it was thus revealed that PARP1 may be a novel candidate drug target in RAD51/RAD54B-deficient cells (45). Chromatin remodeling proteins are often contained within multiprotein complexes (43).

Category 4: Components that are arranged in a single linear pathway. The ATR and Chk1 kinases, critical mediators of the DNA damage response pathway, maintain cell survival and protect cells from replication stress (46). These two kinases are currently the focus of ongoing clinical trials (47). Inhibition of Raf-1 proto-oncogene serine threonine kinase (RAF1) is a synthetic lethal target in KRAS mutant tumor types (48). The ATR-Chk1 and KRAS-RAF1 pathways function as a cancer-specific synthetic lethality between two proteins in the same pathway $(47,48)$.

The present review discussed recent developments in the synthetic lethality gene pairs in four categories, which includes licensed inhibitors in preclinical and clinical trials: i) PARP inhibitors for homologous recombination deficient cancer types; ii) disruption of the ATM paralog ATR; iii) deficiency of BRM or BRG in an essential multiprotein complex and iv) two proteins in the same pathway, ATR and Chk1. Recent clinical studies reported the evidence for the use of PARP inhibitors in high-grade serous ovarian cancer with germline or somatic BRCA1/2 mutations (49).

\section{Synthetic lethal partners in CCC}

Preclinical and clinical data of targeting synthetic lethal partners for the treatment of $\mathrm{CCC}$ cell lines and patients with CCC, respectively, are discussed as follows. Firstly, previous literature suggests a lack of prognostic and early detection markers available for CCC (21). However, Arakawa et al (50) reported that tissue factor pathway inhibitor 2 may be a useful serum marker for the detection of CCC. In addition, the overexpression of transcription factor $\mathrm{HNF}(>90 \%)(17)$ and somatic mutations of ARID1A (15) and PIK3CA ( 50\%) (51) are common genetic changes reported in CCC (52). In previous study, suppression of HNF expression resulted in apoptotic cell death in CCC cell lines, indicating that HNF may be associated with tumor survival (18). Thus, HNF may be suggested as a desirable candidate for targeted cancer therapy. However, the pharmacological inhibition of HNF may be particularly toxic for normal cells as this transcription factor is expressed in the liver, digestive tract, pancreas and kidneys (18). Chk1 has been identified as a downstream target of HNF, demonstrating that the overexpression or persistent phosphorylation of Chk1 is a characteristic feature of the HNF-overexpressing CCC (18). The HNF-Chk1 axis serves a critical role in the cell cycle arrest at G2/M phase and DNA repair (20). CCC is sensitive to Chk1 inhibitors as they induce a high degree of HNF-induced replication stress (22). The HNF-Chk1 axis is a main pathway for cell survival in CCC (20). These data suggest that the inhibition of Chk1 may be lethal in HNF-overexpressing CCC cells.
Secondly, ATR and Chk1 are a synthetic lethal pair of two proteins in the same pathway and maintain cancer cell survival under replication stress (47). Inhibitors of ATR and Chk1 are currently undergoing clinical trials and may represent a promising strategy for cancer therapy (47). Furthermore, a randomized phase II trial study has been conducted to assess whether adding ATR kinase inhibitor M6620 to standard treatment with gemcitabine hydrochloride is more effective than gemcitabine hydrochloride alone in treating patients with ovarian, primary peritoneal or fallopian tube cancer (trial number, NCT02595892) (53). A phase II single arm pilot study has been conducted to determine the objective response rate of single agent Chk1/2 inhibitor (LY2606368) in patients with germline BRCA mutation-associated breast or ovarian cancer, high-grade serous cancer and triple-negative breast cancer (trial number, NCT02203513) (54). The latter study provided positive proof of concept of the efficacy and tolerability of the Chk1/2 inhibitor in patients with ovarian cancer (54). From such studies it may be assumed that ovarian cancer cells, including CCC cells, exhibit an increased reliance on the ATR-Chk1 axis for cell survival.

Thirdly, CCC cells commonly lack compensatory DNA damage response machinery, as mutations in ARID1A represent one of the most common molecular alterations in CCC (15). ARID1A deficiency reportedly triggers genomic instability and apoptosis in ATR-deficient cells (32). These data suggest that ARID1A may be a synthetic lethal partner of ATR.

Finally, the PTEN mutations are also commonly observed in CCC (31). siRNA screening strategies identified PTEN and ATM as synthetically lethal partners (55). One preclinical study demonstrated that ATM inhibition resulted in DNA damage, cell cycle arrest and apoptosis specifically in PTEN-deficient cells (55). PARP inhibition also sensitized cancer cells to genotoxic stress in PTEN-deficient cells (56). Although a variety of loss of function genes, including in HNF, ARID1A, PIK3CA and PTEN, in CCC are driver or passenger events, these genes may function as synthetic lethal pairs under replication stress conditions (16).

\section{Conclusions}

The present article summarized recent advances in molecular understanding of synthetic lethal partners. CCC, the most common type 1 ovarian cancer in Japan, demonstrated chemoresistance resulting in poor prognosis (57). Novel potential targets in CCC therapy were additionally discussed. The development of novel cancer drugs is a time-consuming and expensive process with a high rate of failure in late-stage clinical trials (58). The synthetic lethality strategy to identify novel partners for potential genetic mutations has emerged to overcome this challenge $(5,8)$. Homologous recombination deficiency including BRCA1/2 or p53 mutations combined with an increase in replication stress may result in a tumor being more susceptible to PARP inhibition $(38,45,49,56)$. Since these particular genes are rarely observed to be mutated in CCC, there is considerable interest in identifying alternative therapeutic options of PARP inhibitor sensitivity (2). Synthetic lethal interactions have a variety of types, involving genes that function as a critical backup, paralog genes derived from the 
same ancestral gene, subunit genes of a multiprotein complex or genes that belong to a single linear signaling pathway (8). The present review highlighted promising candidate genes, including p53 and BRCA1-regulated stress-inducible genes and genes involved in the regulation of replication initiation, relevant DNA repair factors and cell cycle distribution, which may be associated with the observed deficiency in homologous recombination capacity. Among these synthetic lethal pairs, the genetic ablation of the DNA repair response genes located in parallel signaling pathways may be one of the important patterns resulting in synthetic lethality. Investigators have unraveled a set of candidate gene targets hypothetically resulting in synthetic lethality. HNF, ARID1A, ATR, ATM, Chk1 and PTEN may be the key genes in CCC $(18,32,47,52,55)$. Notably, the HNF-Chk1 axis was specifically upregulated in CCC (18). ARID1A mutation-driven cancer cells may rely on $\mathrm{HNF}$ overexpression for survival, predicting the potential association between ARID1A and HNF determining synthetic lethality. The pharmacological inhibition of Chk1 is also likely to be synthetically lethal to ARID1A. These predicted genes are promising candidates for in-depth investigation and experimental validation to accumulate significant data for the application of clinical trials. Further clinical studies are required to investigate the safety and therapeutic effects of synthetic lethality pairs in CCC tumor types with replication stress.

In conclusion, the development of synthetic lethal approaches may expedite the formation of novel concepts regarding targeted cancer therapeutics in the future, and this strategy represents a potentially paradigm-shifting initiative. An understanding of high-confidence synthetic lethal interaction pairs may aid in the development of cancer therapies.

\section{Acknowledgements}

Not applicable.

\section{Funding}

The present study was supported by the grant-in-aid for scientific research from the Ministry of Education, Science and Culture of Japan to the Department of Obstetrics and Gynecology, Nara Medical University, Nara, Japan (awarded to Dr Hiroshi Kobayashi; grant no. 26293361).

\section{Availability of data and materials}

Not applicable.

\section{Authors' contributions}

SM collected data regarding the signaling pathways and underlying mechanism of synthetic lethality using the SynLethDB database. KI, EN and SM performed the literature search and supervised the study. YY, KO and NK made substantial contribution to conception of the study. HK contributed to the study design and interpretation of included research studies. The final version of the manuscript has been read and approved by all authors.

\section{Ethics approval and consent to participate}

Not applicable.

\section{Consent for publication}

Not applicable.

\section{Competing interests}

The authors declare that the research was conducted in the absence of any commercial or financial relationships that could be construed as a potential conflict of interest.

\section{References}

1. Siegel RL, Miller KD and Jemal A: Cancer statistics, 2016. CA Cancer J Clin 66: 7-30, 2016.

2. Kurman RJ and Shih IeM: The origin and pathogenesis of epithelial ovarian cancer: A proposed unifying theory. Am J Surg Pathol 34: 433-443, 2010.

3. Kroeger PT Jr and Drapkin R: Pathogenesis and heterogeneity of ovarian cancer. Curr Opin Obstet Gynecol 29: 26-34, 2017.

4. Saijo N: Present status and problems on molecular targeted therapy of cancer. Cancer Res Treat 44: 1-10, 2012.

5. Thompson N, Adams DJ and Ranzani M: Synthetic lethality: Emerging targets and opportunities in melanoma. Pigment Cell Melanoma Res 30: 183-193, 2017.

6. Duan P, Fan L, Gao Q, Silwal BM, Ren M, Shen Y and Qu W: Targeted Therapy of Ovarian Cancer with Angiogenesis Inhibitors. Curr Drug Targets 18: 1171-1178, 2017.

7. Hamanishi J, Mandai M, Ikeda T, Minami M, Kawaguchi A, Murayama T, Kanai M, Mori Y, Matsumoto S, Chikuma S, et al: Safety and Antitumor Activity of Anti-PD-1 Antibody, Nivolumab, in Patients With Platinum-Resistant Ovarian Cancer. J Clin Oncol 33: 4015-4022, 2015.

8. Fang B: Development of synthetic lethality anticancer therapeutics. J Med Chem 57: 7859-7873, 2014.

9. Perets R, Wyant GA, Muto KW, Bijron JG, Poole BB, Chin KT, Chen JY, Ohman AW, Stepule CD, Kwak S, et al: Transformation of the fallopian tube secretory epithelium leads to high-grade serous ovarian cancer in Brca;Tp53;Pten models. Cancer Cell 24: 751-765, 2013.

10. Scully R: Role of BRCA gene dysfunction in breast and ovarian cancer predisposition. Breast Cancer Res 2: 324-330, 2000.

11. Turner N, Tutt A and Ashworth A: Hallmarks of 'BRCAness' in sporadic cancers. Nat Rev Cancer 4: 814-819, 2004.

12. Dominguez-Sola D and Gautier J: MYC and the control of DNA replication.Cold Spring Harb Perspect Med 4: pii: a014423, 2014.

13. Kobayashi H, Shigetomi H and Yoshimoto C: Checkpoint kinase 1 inhibitors as targeted molecular agents for clear cell carcinoma of the ovary. Oncol Lett 10: 571-576, 2015.

14. Lyttle B, Bernardi L and Pavone ME: Ovarian cancer in endometriosis: Clinical and molecular aspects. Minerva Ginecol 66: 155-164, 2014.

15. Wiegand KC, Shah SP, Al-Agha OM, Zhao Y, Tse K, Zeng T, Senz J, McConechy MK, Anglesio MS, Kalloger SE, et al: ARID1A mutations in endometriosis-associated ovarian carcinomas. N Engl J Med 363: 1532-1543, 2010.

16. Jin Y, Li Y and Pan L: The target therapy of ovarian clear cell carcinoma. OncoTargets Ther 7: 1647-1652, 2014.

17. Tsuchiya A, Sakamoto M, Yasuda J, Chuma M, Ohta T, Ohki M, Yasugi T, Taketani Y and Hirohashi S: Expression profiling in ovarian clear cell carcinoma: Identification of hepatocyte nuclear factor-1 beta as a molecular marker and a possible molecular target for therapy of ovarian clear cell carcinoma. Am J Pathol 163: 2503-2512, 2003.

18. Shigetomi H, Sudo T, Shimada K, Uekuri C, Tsuji Y, Kanayama S, Naruse K, Yamada Y, Konishi N and Kobayashi H: Inhibition of cell death and induction of G2 arrest accumulation in human ovarian clear cells by HNF-1 $\beta$ transcription factor: Chemosensitivity is regulated by checkpoint kinase CHK1. Int J Gynecol Cancer 24: 838-843, 2014.

19. Peng ZG, Yao YB, Yang J, Tang YL and Huang X: Mangiferin induces cell cycle arrest at G2/M phase through ATR-Chk1 pathway in HL-60 leukemia cells. Genet Mol Res 14: 4989-5002, 2015. 
20. Ito F, Yoshimoto $C$, Yamada $Y$, Sudo $T$ and Kobayashi $H$ : The HNF-1 $\beta$-USP28-Claspin pathway upregulates DNA damage-induced Chk1 activation in ovarian clear cell carcinoma. Oncotarget 9: 17512-17522, 2018.

21. Kobayashi H, Sugimoto H, Onishi S and Nakano K: Novel biomarker candidates for the diagnosis of ovarian clear cell carcinoma. Oncol Lett 10: 612-618, 2015.

22. Itamochi H, Nishimura M, Oumi N, Kato M, Oishi T, Shimada M, Sato S, Naniwa J, Sato S, Kudoh A, et al: Checkpoint kinase inhibitor AZD7762 overcomes cisplatin resistance in clear cell carcinoma of the ovary. Int J Gynecol Cancer 24: 61-69, 2014.

23. Yang $\mathrm{S}, \mathrm{Wu} \mathrm{L}, \mathrm{Li} \mathrm{X}$, Huang J, Zhong $\mathrm{J}$ and Chen $\mathrm{X}$ : Crizotinib-associated toxic epidermal necrolysis in an ALK-positive advanced NSCLC patient. Mol Clin Oncol 8: 457-459, 2018.

24. Mirza MR, Monk BJ, Herrstedt J, Oza AM, Mahner S, Redondo A,Fabbro M, Ledermann JA, Lorusso D, Vergote I, et al ENGOT-OV16/NOVA Investigators: Niraparib Maintenance Therapy in Platinum-Sensitive, Recurrent Ovarian Cancer. N Engl J Med 375: 2154-2164, 2016.

25. Papa A, Caruso D, Strudel M, Tomao S and Tomao F: Update on Poly-ADP-ribose polymerase inhibition for ovarian cancer treatment. J Transl Med 14: 267, 2016.

26. Farmer H, McCabe N, Lord CJ, Tutt AN, Johnson DA, Richardson TB, Santarosa M, Dillon KJ, Hickson I, Knights C, et al: Targeting the DNA repair defect in BRCA mutant cells as a therapeutic strategy. Nature 434: 917-921, 2005.

27. Raj L, Ide T, Gurkar AU, Foley M, Schenone M, Li X, Tolliday NJ, Golub TR, Carr SA, Shamji AF, et al: Selective killing of cancer cells by a small molecule targeting the stress response to ROS. Nature 475: 231-234, 2011.

28. Guo J, Liu H and Zheng J: SynLethDB: Synthetic lethality database toward discovery of selective and sensitive anticancer drug targets. Nucleic Acids Res 44 (D1): D1011-D1017, 2016.

29. Pathak HB, Zhou Y, Sethi G, Hirst J, Schilder RJ, Golemis EA and Godwin AK: A Synthetic Lethality Screen Using a Focused siRNA Library to Identify Sensitizers to Dasatinib Therapy for the Treatment of Epithelial Ovarian Cancer. PLoS One 10: e0144126, 2015

30. Crespan E, Garbelli A, Amoroso A and Maga G: Exploiting the nucleotide substrate specificity of repair DNA polymerases to develop novel anticancer agents. Molecules 16: 7994-8019, 2011.

31. Bitler BG, Fatkhutdinov $N$ and Zhang R: Potential therapeutic targets in ARID1A-mutated cancers. Expert Opin Ther Targets 19: 1419-1422, 2015.

32. Williamson CT, Miller R, Pemberton HN, Jones SE, Campbell J, Konde A, Badham N, Rafiq R, Brough R, Gulati A, et al: ATR inhibitors as a synthetic lethal therapy for tumours deficient in ARID1A. Nat Commun 7: 13837, 2016.

33. Jdey W, Thierry S, Russo C, Devun F, Al Abo M,Noguiez-Hellin P, Sun JS, Barillot E, Zinovyev A, Kuperstein I, et al: Drug-Driven Synthetic Lethality: Bypassing Tumor Cell Genetics with a Combination of AsiDNA and PARP Inhibitors. Clin Cancer Res 23: 1001-1011, 2017.

34. Toledo LI, Murga M, Zur R, Soria R, Rodriguez A, Martinez S, Oyarzabal J, Pastor J, Bischoff JR and Fernandez-Capetillo O: A cell-based screen identifies ATR inhibitors with synthetic lethal properties for cancer-associated mutations. Nat Struct Mol Biol 18: 721-727, 2011

35. Subhash VV, Tan SH, Yeo MS, Yan FL, Peethala PC, Liem N, Krishnan V and Yong WP: ATM Expression Predicts Veliparib and Irinotecan Sensitivity in Gastric Cancer by Mediating P53-Independent Regulation of Cell Cycle and Apoptosis. Mol Cancer Ther 15: 3087-3096, 2016.

36. Wang X, Zhang Y, Han ZG and He KY: Malignancy of Cancers and Synthetic Lethal Interactions Associated With Mutations of Cancer Driver Genes. Medicine (Baltimore) 95: e2697, 2016.

37. Carvalho JF and Kanaar R: Targeting homologous recombination-mediated DNA repair in cancer. Expert Opin Ther Targets 18: 427-458, 2014.

38. Liu FW and Tewari KS: New Targeted Agents in Gynecologic Cancers: Synthetic Lethality, Homologous Recombination Deficiency, and PARP Inhibitors. Curr Treat Options Oncol 17: $12,2016$.

39. Smith J, Tho LM, Xu N and Gillespie DA: The ATM-Chk2 and ATR-Chk1 pathways in DNA damage signaling and cancer. Adv Cancer Res 108: 73-112, 2010.

40. Jin Q, Yu LR, Wang L, Zhang Z, Kasper LH, Lee JE, Wang C, Brindle PK, Dent SY and Ge K: Distinct roles of GCN5/PCAF-mediated H3K9ac and CBP/p300-mediated $\mathrm{H} 3 \mathrm{~K} 18 / 27 \mathrm{ac}$ in nuclear receptor transactivation. EMBO J 30: 249-262, 2011
41. Ogiwara H, Sasaki M, Mitachi T, Oike T, Higuchi S, Tominaga Y and Kohno T: Targeting p300 Addiction in CBP-Deficient Cancers Causes Synthetic Lethality by Apoptotic Cell Death due to Abrogation of MYC Expression. Cancer Discov 6: 430-445, 2016.

42. Hoffman GR, Rahal R, Buxton F, Xiang K, McAllister G, Frias E, Bagdasarian L, Huber J, Lindeman A, Chen D, et al: Functional epigenetics approach identifies BRM/SMARCA2 as a critical synthetic lethal target in BRG1-deficient cancers. Proc Natl Acad Sci USA 111: 3128-3133, 2014.

43. Raab JR, Runge JS, Spear CC and Magnuson T: Co-regulation of transcription by BRG1 and BRM, two mutually exclusive SWI/SNF ATPase subunits. Epigenetics Chromatin 10: 62, 2017.

44. Chandramouly G, McDevitt S, Sullivan K, Kent T, Luz A, Glickman JF, Andrake M, Skorski T and Pomerantz RT: Small-Molecule Disruption of RAD52 Rings as a Mechanism for Precision Medicine in BRCA-Deficient Cancers. Chem Biol 22: 1491-1504, 2015.

45. Wiegmans AP, Yap PY, Ward A, Lim YC and Khanna KK: Differences in Expression of Key DNA Damage Repair Genes after Epigenetic-Induced BRCAness Dictate Synthetic Lethality with PARP1 Inhibition. Mol Cancer Ther 14: 2321-2331, 2015.

46. Gatei M, Kijas AW, Biard D, Dörk T and Lavin MF: RAD50 phosphorylation promotes ATR downstream signaling and DNA restart following replication stress. Hum Mol Genet 23: 4232-4248, 2014

47. Sanjiv K, Hagenkort A, Calderón-Montaño JM, Koolmeister T, Reaper PM, Mortusewicz O, Jacques SA, Kuiper RV, Schultz N, Scobie M, et al: Cancer-Specific Synthetic Lethality between ATR and CHK1 Kinase Activities. Cell Reports 14: 298-309, 2016.

48. Lamba S, Russo M, Sun C,Lazzari L, Cancelliere C, Grernrum W, Lieftink C, Bernards R, Di Nicolantonio F and Bardelli A: RAF suppression synergizes with MEK inhibition in KRAS mutant cancer cells. Cell Reports 8: 1475-1483, 2014.

49. Morgan RD, Clamp AR, Evans DGR, Edmondson RJ and Jayson GC: PARP inhibitors in platinum-sensitive high-grade serous ovarian cancer. Cancer Chemother Pharmacol 81: 647-658, 2018.

50. Arakawa N, Kobayashi H, Yonemoto N, Masuishi Y, Ino Y, Shigetomi H, Furukawa N, Ohtake N, Miyagi Y, Hirahara F, et al: Clinical Significance of Tissue Factor Pathway Inhibitor 2, a Serum Biomarker Candidate for Ovarian Clear Cell Carcinoma. PLoS One 11: e0165609, 2016.

51. Wang Y, Helland A, Holm R, Kristensen GB and Børresen-Dale AL: PIK3CA mutations in advanced ovarian carcinomas. Hum Mutat 25: 322, 2005.

52. Matsuzaki S, Yoshino K, Ueda Y, Matsuzaki S, Kakuda M, Okazawa A, Egawa-Takata T, Kobayashi E and Kimura T: Potential targets for ovarian clear cell carcinoma: A review of updates and future perspectives. Cancer Cell Int 15: 117, 2015.

53. Fokas E, Prevo R, Pollard JR, Reaper PM, Charlton PA, Cornelissen B, Vallis KA, Hammond EM, Olcina MM, Gillies McKenna W, et al: Targeting ATR in vivo using the novel inhibitor VE-822 results in selective sensitization of pancreatic tumors to radiation. Cell Death Dis 3: e441, 2012.

54. Lee JM, Nair J, Zimmer A, Lipkowitz S, Annunziata CM, Merino MJ, Swisher EM, Harrell MI, Trepel JB, Lee MJ, et al: Prexasertib, a cell cycle checkpoint kinase 1 and 2 inhibitor, in BRCA wild-type recurrent high-grade serous ovarian cancer: A first-in-class proof-of-concept phase 2 study. Lancet Oncol 19: 207-215, 2018.

55. McCabe N, Hanna C, Walker SM, Gonda D, Li J, Wikstrom K, Savage KI, Butterworth KT, Chen C, Harkin DP, et al: Mechanistic Rationale to Target PTEN-Deficient Tumor Cells with Inhibitors of the DNA Damage Response Kinase ATM. Cancer Res 75: 2159-2165, 2015.

56. Dedes KJ, Wetterskog D, Mendes-Pereira AM, Natrajan R, Lambros MB, Geyer FC, Vatcheva R, Savage K, Mackay A, Lord CJ, et al: PTEN deficiency in endometrioid endometrial adenocarcinomas predicts sensitivity to PARP inhibitors. Sci Transl Med 2: 53ra75, 2010.

57. Sugiyama T, Kamura T, Kigawa J, Terakawa N, Kikuchi Y, Kita T, Suzuki M, Sato I and Taguchi K: Clinical characteristics of clear cell carcinoma of the ovary: A distinct histologic type with poor prognosis and resistance to platinum-based chemotherapy. Cancer 88: 2584-2589, 2000.

58. Basith S, Cui M, Macalino SJY and Choi S: Expediting the Design, Discovery and Development of Anticancer Drugs using Computational Approaches. Curr Med Chem 24: 4753-4778, 2017. 